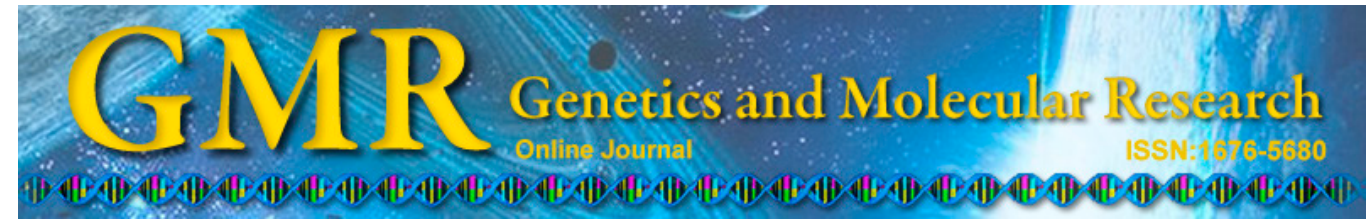

\title{
Safety of recombinant human granulocyte- macrophage colony-stimulating factor in healing pediatric severe burns
}

\author{
Y.F. Chi, J.K. Chai, H.M. Luo, Q.X. Zhang and R. Feng \\ Department of Burns and Plastic Surgery, \\ First Hospital Affiliated to The Peoples Liberation Army General Hospital, \\ Beijing, China \\ Corresponding author: Y.F. Chi \\ E-mail: chiyunfei_cyf@163.com
}

Genet. Mol. Res. 14 (1): 2735-2741 (2015)

Received November 3, 2014

Accepted March 18, 2015

Published March 31, 2015

DOI http://dx.doi.org/10.4238/2015.March.31.3

\begin{abstract}
We explored the safety of recombinant human granulocyte-macrophage colony-stimulating factor (rhGM-CSF) for healing burns in children. Subjects were randomly assigned to two groups: the experimental group received external rhGM-CSF gel, and the control group received rhGM-CSF gel matrix components, applied to the burn surface. Neither group was given any other drugs that promote wound healing. Each day we recorded the pulse, body temperature, and respiration status in the two groups. We detected the blood routine, urine routine, and hepatic and renal function before the patients received drug treatment and after $72 \mathrm{~h}$. The wound scab and healing states in the two groups were recorded every 4 days to evaluate wound healing rate and time taken for complete healing. Adverse reactions and their rate of occurrence were also recorded. The median time of healing was 15 days in the experimental group and 19 days in the control group (log-rank $\chi^{2}=$ $5.139, \mathrm{P}<0.05)$. After 10 days, the experimental group healing rate was consistently higher than that of the control group (significantly different using intuitive analysis), suggesting the experimental group method was more effective. There were no obvious adverse reactions. There was no
\end{abstract}


significant difference between the blood routine, urine routine, and liver and kidney function in the two groups before the treatment and after 3 days $(\mathrm{P}>0.05)$. Compared with saline treatment of severe burns, rhGMCSF can effectively shorten the healing time without significant adverse reactions, and is an effective and safe treatment for burns in children.

Key words: rhGM-CSF; Children; Severe burns; Safety; Recombinant human granulocyte-macrophage colony-stimulating factor

\section{INTRODUCTION}

Burns are a common surgical affliction with a high incidence rate caused mainly by heat. They are common in daily life and are more prevalent in children between the ages of 1 and 5 years (Shi, 2012). The degree of physiological development is lower in children and their connective tissue is immature. Therefore, children respond strongly to external adverse reactions and stimulation, and their self-protection capability is poor. When burns occur in children they are usually severe (Zhao et al., 2013). Severe burns are usually repaired by the residual skin appendages of the dermis, which may result in various problems in the healing process and affect the healing rate and quality. In recent years, recombinant human granulocyte-macrophage colony-stimulating factor (rhGM-CSF) has been increasingly valued in healing burns to the skin (Zhang et al., 2009; Zeng, 2012). Although the effectiveness of rhGM-CSF treatment has been confirmed by animal experiments and clinical trials (Liu, 2011; Yang, 2011; Ding, 2012; Yan and Qiu, 2013), clinical safety analysis of rhGM-CSF is lacking, and the results are questionable. Therefore, we conducted a comprehensive safety analysis to clarify the clinical implications of rhGM-CSF in the healing of pediatric severe burns.

\section{MATERIAL AND METHODS}

\section{Subjects}

We selected 30 children with burns in our hospital between December 2013 and June 2014. Their ages ranged from 1 to 5 years and the mean age was $3.25 \pm 1.08$ years; there were 16 males and 14 females. There were 21 cases of burns caused by heat and 9 cases caused by naked flames. Inclusion criteria were: that the children's burn areas were severe (20-25\%); the children had received no other treatment before admission; and that they had no severe allergies or other serious diseases. The exclusion criteria were: that the children had received other treatment before admission; and that they had serious heart disease, unstable angina, heart failure, myocardial infarction, or systemic infection. Each child's parents signed "informed consent" developed by our medical ethics institute.

\section{Methods}

All patients were given $1 \%$ povidone-iodine for routine debridement and disinfection. After physiological saline cleaning, amikacin was uniformly sprayed on the wounds. The patients were randomly divided into two groups. The experimental group $(\mathrm{N}=15)$ was treated with external rhGM-CSF gel (specification: each 100 mg: 10 g; State Medical Permit S20080003; 
Changchun Jinsai Pharmaceutical Co. Ltd.). A slightly larger sterilization baseline gauze was used to cover burns after dressing, followed by the main gauze. The dressings were changed daily until the wounds healed. The control group $(\mathrm{N}=15)$ was treated with the rhGM-CSF gel matrix components (Changchun Jinsai Pharmaceutical Co. Ltd.). Neither group was given any other drugs that promote wound healing. If wound infection occurred, systemic antibiotics were used to control the infection. We recorded and analyzed wound healing states in the two groups.

\section{Observation indices}

We made daily recordings of the pulse, body temperature, and respiration status of the children in the two groups. We also recorded the blood routine, urine routine, and hepatic and renal function state before the patients received drug treatment and after $72 \mathrm{~h}$. The wound scab and healing states of the children in the two groups were recorded every 8 days to evaluate wound healing rate and time taken for complete healing. Adverse reactions (such as fever, pain, local swelling, rashes, and itching) were recorded and their rate of incidence was also calculated. If serious adverse reactions occurred, measures were taken to protect the safety of the subjects in a timely manner.

$$
\begin{gathered}
\begin{array}{c}
\text { Wound healing rate }=[(\text { area of pre-treatment })-(\text { area of post-treatment })] / \\
(\text { area of pre-treatment }) \times 100 \%
\end{array} \\
\text { Incidence of adverse reaction }=\text { (number of adverse reaction cases }) / \\
\text { (total number of cases) } \times 100 \%
\end{gathered}
$$

\section{Statistical analysis}

All data were analyzed using the SPSS17.0 statistical software. The wound healing times in the two groups were compared using the log-rank method. We compared the wound healing rate in the two groups using the Wilcoxon rank sum test. Other indices in the two groups were compared using the Student $t$-test, and the data are reported as means $\pm \mathrm{SD}$. $\mathrm{P}$ values $<0.05$ were considered to be statistically significant.

\section{RESULTS}

Figure 1 compares burns in children before and after treatment.
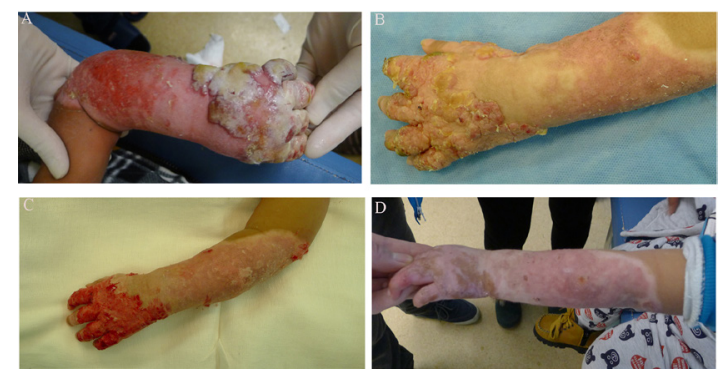

Figure 1. Comparison chart showing burns in children before and after treatment. A. Condition on the day burn occurred. B. Degree of healing after treatment for 8 days. C. Degree of healing after treatment for 16 days. D. Degree of healing after treatment for 24 days. 


\section{Wound healing time and rate}

The median wound healing time was 15 days in the experimental group and 19 days in the control group; the difference between the two groups was statistically significant according to the log-rank method $\left(\log\right.$-rank $\left.\chi^{2}=5.139, \mathrm{P}<0.05\right)$. After 10 days, the wound healing rate in the experimental group was consistently higher than in the control group; the differences between the two groups were significant according to intuitive analysis, which suggests that the method used in the experimental group was more effective. The results are shown in Figure 2.

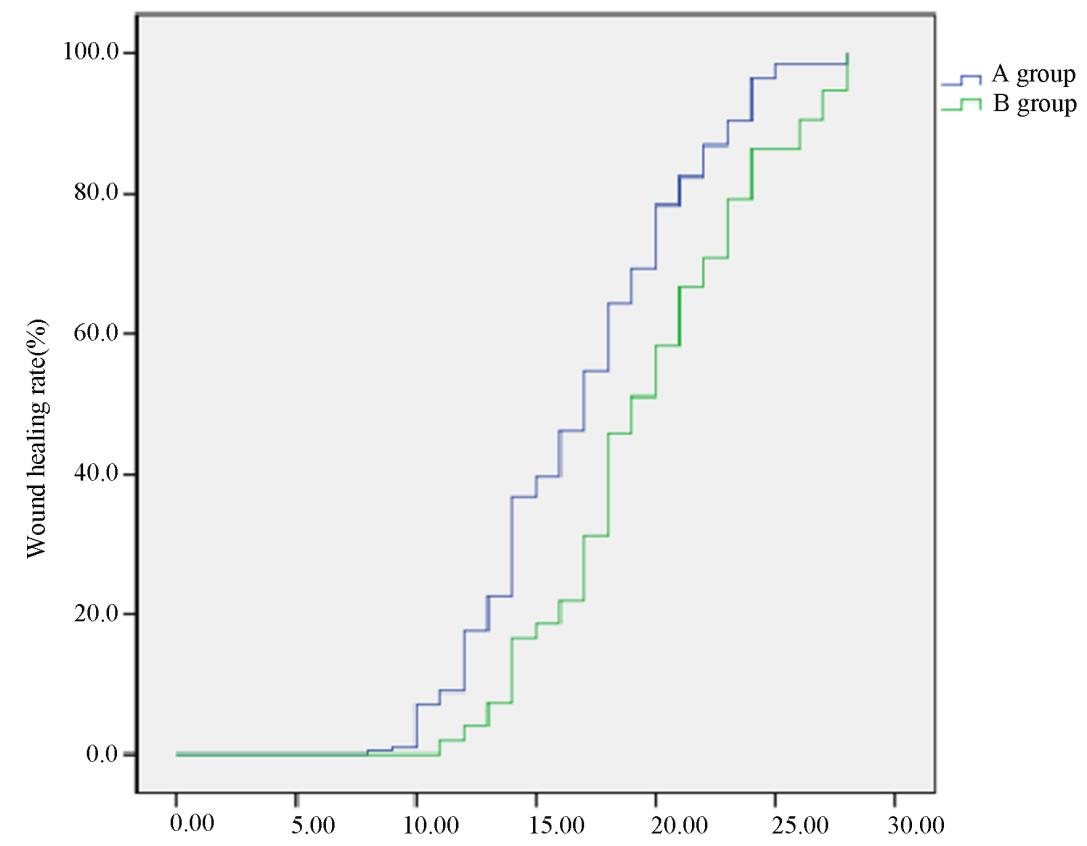

Figure 2. Statistical analysis curve of wound healing rate versus time in the two groups.

\section{Incidence of adverse reactions}

There were no obvious adverse reaction in either group, but three patients in the experimental group experienced fever, runny noses, and other symptoms during the treatment process, which were ultimately determined to be upper respiratory tract infections. One child experienced diarrhea, which was ultimately identified as symptomatic diarrhea. The symptoms improved after 3-5 days. Because the symptoms experienced by these four children were not associated with the test drugs, the incidence rate of adverse reactions was taken to be zero.

\section{Safety analysis (blood routine, urine routine, and liver and kidney function detection results)}

There were no statistically significant differences in the blood routine tests (white blood cells, neutrophil percentage, hemoglobin, and platelets) between the two groups ( $\mathrm{P}>$ 
0.05 ) (the normal white blood cell count in children is 4.0-10.0 x 10\%/L; the normal neutrophil percentage value is $0.33-0.79$; the normal hemoglobin concentration is $110-160 \mathrm{~g} / \mathrm{L}$; and the normal platelet count is $100-300 \times 10^{9} / \mathrm{L}$ ).

Detection of the urine routine in the two groups (the specific gravity and $\mathrm{pH}$ of the urine) did not reveal abnormal red blood cell or protein levels, and the differences in urine specific gravity and $\mathrm{pH}$ in the two groups were not statistically significant $(\mathrm{P}>0.05)$ (the normal specific gravity of urine in children is 1.003-1.030 and the normal urine $\mathrm{pH}$ is 4.6-8.0).

Liver and kidney function was detected in the two groups [total bilirubin, total protein, albumin, alanine aminotransferase (ALT); aspartate transaminase (AST), creatinine, and urea nitrogen]. There were no significant differences in total bilirubin, total protein, albumin, ALT, AST, creatinine, or urea nitrogen in the two groups $(\mathrm{P}>0.05)$ (the normal concentration of children's total bilirubin is $1.8-21 \mu \mathrm{M}$; the normal concentration of total protein is $60-83 \mathrm{~g} / \mathrm{L}$; the normal concentration of albumin is $35-55 \mathrm{~g} / \mathrm{L}$; the normal concentration of ALT is $0-50$ $\mathrm{U} / \mathrm{L}$; the normal concentration of AST is $0-50 \mathrm{U} / \mathrm{L}$ : the normal concentration of creatinine is 14.4-88.4 $\mu \mathrm{M}$; and the normal concentration of urea nitrogen is $2.87-7.14 \mathrm{mM}$ ). The results are shown in Tables 1, 2, and 3 .

Table 1. Comparison of the blood routines of the children in the two groups (means $\pm \mathrm{SD}$ ).

\begin{tabular}{|c|c|c|c|c|c|c|c|c|}
\hline \multirow[t]{2}{*}{ Group } & \multicolumn{2}{|c|}{ WBC (x $\left.10^{9} / \mathrm{L}\right)$} & \multicolumn{2}{|c|}{ N (\%) } & \multicolumn{2}{|c|}{$\mathrm{Hb}(\mathrm{g} / \mathrm{L})$} & \multicolumn{2}{|c|}{$\operatorname{PLT}\left(\times 10^{9} / \mathrm{L}\right)$} \\
\hline & $0 \mathrm{~h}$ & $72 \mathrm{~h}$ & $0 \mathrm{~h}$ & $72 \mathrm{~h}$ & $0 \mathrm{~h}$ & $72 \mathrm{~h}$ & $0 \mathrm{~h}$ & $72 \mathrm{~h}$ \\
\hline Test group & $9.96 \pm 2.45$ & $9.09 \pm 1.78$ & $0.44 \pm 0.10$ & $0.45 \pm 0.14$ & $116 \pm 9$ & $118 \pm 10$ & $296 \pm 98$ & $296 \pm 57$ \\
\hline Control group & $9.79 \pm 2.66$ & $9.01 \pm 1.47$ & $0.38 \pm 0.12$ & $0.42 \pm 0.09$ & $120 \pm 9$ & $121 \pm 8$ & $270 \pm 78$ & $276 \pm 69$ \\
\hline$t$-value & 0.542 & 1.112 & 1.345 & 0.236 & 1.509 & 2.007 & 0.436 & 0.457 \\
\hline$P$ value & $>0.05$ & $>0.05$ & $>0.05$ & $>0.05$ & $>0.05$ & $>0.05$ & $>0.05$ & $>0.05$ \\
\hline
\end{tabular}

$\mathrm{WBC}=$ white blood cells; $\mathrm{N}=$ neutrophils; $\mathrm{Hb}=$ hemoglobin; $\mathrm{PLT}=$ platelets.

Table 2. Comparison of the urine routines of the children in the two groups (means $\pm \mathrm{SD}$ ).

\begin{tabular}{lrcr}
\hline & & Specific gravity & Urinary pH \\
\hline Test group & $0 \mathrm{~h}$ & $1.011 \pm 0.003$ & $6.4 \pm 0.78$ \\
& $72 \mathrm{~h}$ & $1.017 \pm 0.006$ & $5.6 \pm 0.69$ \\
Control group & $0 \mathrm{~h}$ & $1.014 \pm 0.004$ & $6.0 \pm 0.52$ \\
& $72 \mathrm{~h}$ & $1.015 \pm 0.005$ & $6.1 \pm 0.59$ \\
$t$-value & & 1.178 & 0.045 \\
P value & & $>0.05$ & $>0.05$ \\
\hline
\end{tabular}

Table 3. Comparison of the liver and kidney function of the children in the two groups (means $\pm \mathrm{SD}$ ).

\begin{tabular}{lrccccccc}
\hline Group & & TBIL $(\mu \mathrm{M})$ & TP $(\mathrm{g} / \mathrm{L})$ & Alb $(\mathrm{g} / \mathrm{L})$ & ALT $(\mathrm{U} / \mathrm{L})$ & AST $(\mathrm{U} / \mathrm{L})$ & Cr $(\mu \mathrm{M})$ & BUN $(\mathrm{mM})$ \\
\hline Test group & $0 \mathrm{~h}$ & $7.3 \pm 3.2$ & $62.1 \pm 4.8$ & $44.8 \pm 2.9$ & $14.6 \pm 2.9$ & $37.4 \pm 2.5$ & $19.8 \pm 10.5$ & $3.51 \pm 0.44$ \\
& $72 \mathrm{~h}$ & $6.8 \pm 2.9$ & $62.9 \pm 5.2$ & $44.8 \pm 2.7$ & $15.1 \pm 3.1$ & $30.5 \pm 2.9$ & $16.4 \pm 9.4$ & $4.12 \pm 0.57$ \\
Control group & $0 \mathrm{~h}$ & $9.6 \pm 3.6$ & $60.9 \pm 4.5$ & $45.1 \pm 2.0$ & $21.1 \pm 3.9$ & $38.0 \pm 3.5$ & $20.5 \pm 9.8$ & $4.09 \pm 0.32$ \\
& $72 \mathrm{~h}$ & $10.4 \pm 4.2$ & $65.4 \pm 4.3$ & $45.9 \pm 2.3$ & $22.5 \pm 4.1$ & $38.9 \pm 3.7$ & $21.3 \pm 10.9$ & $4.12 \pm 0.87$ \\
$t$-value & & 1.294 & 0.043 & 2.533 & 1.045 & 0.345 & 0.114 & 0.042 \\
P value & $>0.05$ & $>0.05$ & $>0.05$ & $>0.05$ & $>0.05$ & $>0.05$ & $>0.05$ \\
\hline
\end{tabular}

$\mathrm{TBIL}=$ total bilirubin; $\mathrm{TP}=$ total protein; $\mathrm{Alb}=$ albumin; ALT $=$ alanine aminotransferase; AST $=$ aspartate transaminase; $\mathrm{Cr}=$ creatinine; $\mathrm{BUN}=$ blood urea nitrogen. 


\section{DISCUSSION}

Wound healing is a complex physiological process that involves a variety of growth factors, cytokines, inflammatory cells, and repair cells. Neutrophils are the first participants in cell repair, but they have a short life expectancy and are eventually engulfed by macrophages, which adjust their function to play a dominant role in the process of wound healing by effecting major repairs. rhGM-CSF can cause the release of various growth factors that promote wound healing through the activation of macrophages, and can directly stimulate the migration and differentiation of the stratum corneum cells of the wound surface. This is conducive to the movement of epithelial cells and can promote the re-epithelialization of proliferation cells. In this study, rhGM-CSF systematically repaired damaged skin effectively, accelerated the healing rate, shortened the healing time, and remodeled the connective tissue of the skin. The results were consistent with those of Liu et al. (2010) and Yan et al. (2012).

This study investigated severe burns in patients aged 1-5 years, which is an active period for children. They also have a strong thirst for knowledge and excessive curiosity. However, the spinal growth in small children is incomplete, which means they have poor coordination and slow reaction speeds. Moreover, such young children are still developing intellectually, are less aware of potential danger, and have an underdeveloped sense of self-preservation. Unfortunately, some parents have a low sense of responsibility with regards to their child's safety, which may result in accidental burns or scalds. When a burn occurs in a child, it is often more severe than in an adult because the child's stratum corneum is thin and their resistance to external stimuli is poor (Li et al., 2010; Wu and Li, 2010; Zhang and Kong, 2010; Kang and Yu, 2011).

In this study, we found that the healing time was shorter, the healing rate was higher, and the clinical results were better when the child's burns were treated with external rhGM-CSF gel. These results are consistent with those of Wang (2014). We found that the adverse reaction rate arising from the use of external rhGM-CSF gel was low. When we compared the blood routine (white blood cells, neutrophil percentage, hemoglobin, and platelets), the urine routine (urine specific gravity and $\mathrm{pH}$ ), and liver and kidney function (total bilirubin, total protein, albumin, ALT, AST, creatinine, and blood urea nitrogen) in children before treatment and after 3 days, we found that the topical rhGM-CSF gel produced no health hazards and was clinically safe.

Although the results are satisfactory, the study has some limitations, namely a deficiency in the number of cases and a short period of observation. To ensure the reliability of the results, a larger study sample is required.

\section{Conflicts of interest}

The authors declare no conflict of interest.

\section{REFERENCES}

Ding XB (2012). The effect of recombinant human granulocyte macrophage colony stimulating factor gel on the healing of deep II degree burn wound in Rats. Journal of Luzhou Medical College, Luzhou.

Kang QJ and Yu QH (2011). The causes and clinical features of pediatric burn. Nei Mongol. J. Trad. Chin. Med. 30: 48.

Li BB, Bai M and Yang Y (2010). The research progress of recombinant human granulocyte macrophage colony stimulating factor. Heilongjiang Pharm. 23: 407-408.

Liu B, Yang YH, Wu ZG, et al. (2010). The different doses of rhGM-CSF gel on the healing of deep II degree burn wound in rats. Infect. Inflamm. Repair 11: 71-75. 
Liu JS (2011). The mechanism of GM-CSF gel on deep II degree burn wound eschar. Journal of Bengbu Medical College, Anhui.

Shi CW (2012). Recombinant human granulocyte macrophage colony stimulating factor gel in the treatment of 55 residual burn wounds. Shaanxi Med. J. 41: 882-888.

Wang H (2014). The study of recombinant human granulocyte macrophage colony stimulating factor on the healing of facial deep II degree burn wound in children. Journal of Jilin University, Jilin.

Wu XF and Li W (2010). Analysis of risk factors of pediatric burn. Chin. J. Clin. Med. 8: 586.

Yan H, Chen J and Peng X (2012). Recombinant human granulocyte-macrophage colony-stimulating factor hydrogel promotes healing of deep partial thickness burn wounds. Burns 38: 877-881.

Yan XH and Qiu L (2013). Clinical comparison of rhGM-CSF and rb-bFGF in the treatment of small area burn wound in children. J. Pediatr. Pharm. 19: 11-14.

Yang JF (2011). Clinical study of granulocyte-macrophage colony stimulating factor on the treatment of residual burn wound. Med. Rev. 15: 1554-1556.

Zeng JD (2012). Clinical study of external recombinant human granulocyte macrophage colony stimulating factor gel in the treatment of deep II degree burn wound. Journal of Luzhou Medical College, Luzhou.

Zhang L, Chen J and Han C (2009). A multicenter clinical trial of recombinant human GM-CSF hydrogel for the treatment of deep second-degree burns. Wound Repair Regen. 17: 685-689.

Zhang XJ and Kong NQ (2010). The efficacy of recombinant human granulocyte colony stimulating factor in the treatment of malignant tumor after chemotherapy. China Pharm. 21: 713-715.

Zhao HN, Wang Z, Li HC, et al. (2013). The spray preparation and pharmacodynamic study of recombinant human granulocyte macrophage colony stimulating factor. Northwest Pharmaceut. J. 28: 72-74. 